\title{
交流電場下における片持ち超磁歪／圧電積層材料の動的曲げ Dynamic Bending of Clamped-Free Giant Magnetostrictive/Piezoelectric Laminates under AC Electric Fields

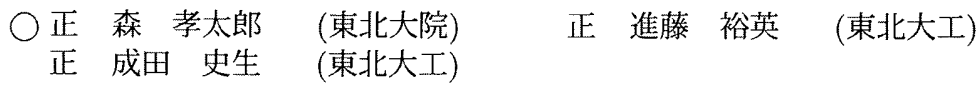

Kotaro MORI, Tohoku University, Aoba-yama 6-6-02, Sendai 980-8579

Yasuhide SHINDO, Tohoku University

Fumio NARITA, Tohoku University

Key Words: Electromagneto-solid mechanics, Finite element method, Material testing, Electronic laminates, Smart materials and structures, Dynamic bending

\section{Introduction}

Magnetoelectric (ME) effect is defined as the polarization of a material in an external magnetic field (direct ME effect), or conversely the magnetization of a material in an external electric field (converse ME effect). The ME materials are likely candidates for magnetic field sensors, memory devices, and electric or magnetic field controlled signal prosessing devices. A great $\mathrm{ME}$ effect can be obtained by the laminated composites consisting of piezoelectric $\mathrm{Pb}(\mathrm{Zr}, \mathrm{Ti}) \mathrm{O}_{3}(\mathrm{PZT})$ and giant magnetostrictive $\mathrm{Tb}_{0.3} \mathrm{Dy}_{0.7} \mathrm{Fe}_{2}$ (Terfenol-D) layers. Though many researches on the direct $\mathrm{ME}$ effect of the magnetostrictive/piezoelectric laminates are found in the literature, the investigations on the characterization of the converse $\mathrm{ME}$ effect are very few. In this work, we study the dynamic bending behavior of clamped-free giant magnetostrictive/piezoelectric laminates under AC electric fields in a combined numerical and experimental approach.

\section{Analysis}

The basic equations for magnetostrictive and piezoelectric materials are outlined here. Consider the rectangular Cartesian coordinate system O- $x_{1} x_{2} x_{3}$. The Newton's second law (the equations of motion), and the electric and magnetic Gauss' laws are given by

$$
\begin{gathered}
\sigma_{j i, j}=\rho u_{i, t t} \\
B_{i, i}=0 \\
D_{i, i}=0
\end{gathered}
$$

where $\sigma_{i j}$ is the stress tensor, $B_{i}$ is the magnetic induction vector, $D_{i}$ is the electric displacement vector, $u_{i}$ is the displacement vector, $\rho$ is the mass density, a comma followed by an index denotes partial differentiation with respect to the space coordinate $x_{i}$ or the time $t$, and the summation convention for repeated tensor indices is applied. The constitutive laws are given as follows:

$$
\begin{aligned}
\varepsilon_{i j} & =s_{i j k l}^{H} \sigma_{k l}+d_{k i j}^{\prime} H_{k} \\
B_{i} & =d_{i k l}^{\prime} \sigma_{k l}+\mu_{i k} H_{k}
\end{aligned}
$$

for the magnetostrictive material, and

$$
\begin{aligned}
\varepsilon_{i j} & =s_{i j k l}^{E} \sigma_{k l}+d_{k i j} E_{k} \\
D_{i} & =d_{i k l} \sigma_{k l}+\epsilon_{i k}^{T} E_{k}
\end{aligned}
$$

for the piezoelectric material. Here, $\varepsilon_{i j}$ is the strain tensor, $H_{i}$ is the magnetic field intensity vector, $E_{i}$ is the electric field intensity vector, $s_{i j k l}^{H}, d_{k i j}^{\prime}, \mu_{i j}$ are the constant magnetic field elastic compliance, magnetoelastic constant and magnetic permittivity of magnetostrictive material, and $s_{i j k l}^{E}, d_{k i j}, \epsilon_{i j}^{T}$ are the constant electric field elastic compliance, direct piezoelectric constant and dielectric permittivity

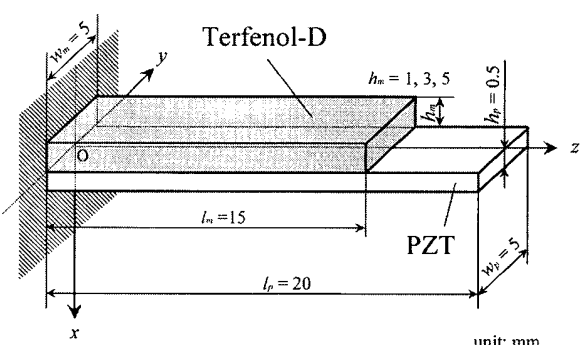

(a)

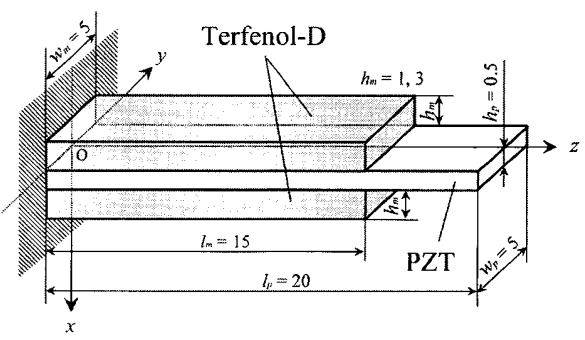

(b)

Fig. 1 Illustration of (a) two-layered and (b) three-layered magnetostrictive/piezoelectric laminate configurations

of piezoelectric material. The relation between the strain tensor and the displacement vector is given by

$$
\varepsilon_{i j}=\frac{1}{2}\left(u_{j, i}+u_{i, j}\right)
$$

The magnetic and electric field intensities are written as

$$
\begin{gathered}
H_{i}=\varphi_{, i} \\
E_{i}=-\phi_{, i}
\end{gathered}
$$

where $\varphi$ and $\phi$ are the magnetic and electric potentials, respectively.

A two-layered magnetostrictive/piezoelectric laminate is shown in Fig. 1(a), in which a magnetostrictive layer, Terfenol-D, of length $l_{m}$, width $w_{m}$ and thickness $h_{m}$ is perfectly bonded on the top surface of a piezoelectric layer, soft PZT, of length $l_{p}$, width $w_{p}$ and thickness $h_{p}$. Dimensions $h_{m}\left(h_{p}\right), w_{m}\left(w_{p}\right), l_{m}\left(l_{p}\right)$ are measured along the $x_{1}=x, x_{2}$ $=y$ and $x_{3}=z$ axis, respectively. The origin of the coordinate system is located at the center of the bottom left side of upper Terfenol-D layer, and the left end $z=0$ is clamped. A three-layered magnetostrictive/piezoelectric laminate is also considered (see Fig. 1(b)). Easy axis of the magnetization of Terfenol-D layer is the $z$-direction, while the polarization of PZT layer is the $x$-direction. 
As we know, nonlinearity of magnetostriction vs magnetic filed curves arises from the rotation of magnetic domains. A magnetic domain switching gives rise to the changes of the magnetoelastic constants, and the constants $d_{15}^{\prime}, d_{31}^{\prime}$ and $d_{33}^{\prime}$ for Terfenol-D layer are

$$
d_{15}^{\prime}=d_{15}^{m}, d_{31}^{\prime}=d_{31}^{m}, d_{33}^{\prime}=d_{33}^{m}+m_{33} H_{z}
$$

where $d_{15}^{m}, d_{31}^{m}, d_{33}^{m}$ are the piezo-magnetic constants, and $m_{33}$ is the second-order magnetoelastic constant. The constant $m_{33}$ can predict well the nonlinearity, without complex computation and more parameters.

On the other hand, domain wall displacement in the piezoelectric materials gives rise to changes of the strain and polarization. For simplicity here, the applied AC electric field $E_{x}=E_{0} \exp (\mathrm{i} \omega t)$ is parallel to the direction of spontaneous polarization $P^{s}$ in one of the domains; $E_{0}$ is the $\mathrm{AC}$ electric field amplitude and $\omega$ is the input frequency. The induced strain $\Delta \varepsilon_{z z}$ and polarization $\Delta P_{x}$ caused by the domain wall motion are given by

$$
\begin{gathered}
\Delta \varepsilon_{z z}=\Delta s_{11}^{E} \sigma_{z z}+\Delta d_{311} E_{x} \\
\Delta P_{x}=\Delta d_{311} \sigma_{z z}+\Delta \epsilon_{33}^{\mathrm{T}} E_{x}
\end{gathered}
$$

where

$$
\Delta s_{11}^{E}=\frac{\gamma^{\mathrm{s} 2}}{2 l f_{\mathrm{D}}}, \Delta d_{311}=-\frac{\gamma^{\mathrm{s}} P^{\mathrm{s}}}{2 l f_{\mathrm{D}}}, \Delta \epsilon_{33}^{\mathrm{T}}=\frac{P^{\mathrm{s} 2}}{2 l f_{\mathrm{D}}}
$$

In Eq. (14), $\gamma^{\mathrm{s}}$ is the spontaneous strain, $l$ is the domain width and $f_{\mathrm{D}}$ represents the force constant for the domain wall motion process. The strain $\varepsilon_{z z}$ and electric displacement $D_{x}$ become

$$
\begin{aligned}
& \varepsilon_{z z}=s_{13}^{E} \sigma_{x x}+s_{12}^{E} \sigma_{y y}+s_{11}^{E *} \sigma_{z z}+d_{31}^{*} E_{x} \\
& D_{x}=d_{33} \sigma_{x x}+d_{31}^{*} \sigma_{y y}+d_{31}^{*} \sigma_{z z}+\epsilon_{33}^{\mathrm{T} *} E_{x}
\end{aligned}
$$

where

$$
s_{11}^{E *}=s_{11}^{E}+\Delta s_{11}^{E}, d_{31}^{*}=d_{31}+\Delta d_{311}, \epsilon_{33}^{\mathrm{T} *}=\epsilon_{33}^{\mathrm{T}}+\Delta \epsilon_{33}^{\mathrm{T}}
$$

The extrinsic dielectric constant $\Delta \epsilon_{33}^{T}$ is described by ${ }^{1)}$

$$
\Delta \epsilon_{33}^{\mathrm{T}}=\epsilon_{33}^{\mathrm{T}} \frac{2 E_{0}}{3 E_{\mathrm{c}}}
$$

where $E_{c}$ is a coercive electric field.

We performed finite element calculations to obtain the displacement, stress and induced magnetic field for TerfenolD/PZT laminates. The basic equations for the magnetostrictive materials are mathematically equivalent to those for piezoelectric materials. So coupled-field solid elements in ANSYS were used in the analysis, and $P^{\mathrm{s}}=0.3 \mathrm{Cm}^{-2}$ and $\gamma^{\mathrm{s}}=0.004$ were assumed to get $\Delta s_{11}^{E}, \Delta d_{311}$ and $\Delta \epsilon_{33}^{\mathrm{T}}$.

\section{Experimental Procedure}

Terfenol-D of $l_{m}=15 \mathrm{~mm}, w_{m}=5 \mathrm{~mm}$ and $h_{m}=1,3,5$ $\mathrm{mm}$ and PZT C-91 of $l_{p}=20 \mathrm{~mm}, w_{p}=5 \mathrm{~mm}$ and $h_{p}=0.5$ $\mathrm{mm}$ were used to make giant magnetostrictive/piezoelectric laminates by epoxy bonding. The second-order magnetoelastic constants $m_{33}$ of Terfenol-D layer with $h_{m}=1,3,5$ $\mathrm{mm}$ of two-layered laminate are $5.0 \times 10^{-12}, 3.3 \times 10^{-12}, 1.0$ $\times 10^{-13} \mathrm{~m}^{2} / \mathrm{A}^{22)}$, and the constants $m_{33}$ of $h_{m}=1,3 \mathrm{~mm}$ of three-layered laminate are $5.2 \times 10^{-12}, 2.3 \times 10^{-12} 3$ ), respectively. The coercive electric field of C-91 is about 0.35 $\mathrm{MV} / \mathrm{m}^{1)}$.
Consider magnetostrictive/piezoelectric laminates driven by $\mathrm{AC}$ voltage with amplitude $V_{0}, V_{0} \exp (\mathrm{i} \omega t)$. AC voltage was applied to the $x=0$ plane of PZT layer, whereas the $x=h_{p}$ plane was grounded. First, the displacement for the two-layered and three-layered laminates under AC voltage was measured with a laser displacement meter. Next, the induced magnetic field of these laminates was obtained from the generated voltage in a search coil with a data logger.

\section{Results and Discussion}

Fig. 2 shows the tip deflection $w_{\text {tip }}$ versus applied AC electric field amplitude $E_{0}=V_{0} / h_{p}$ at $x=y=0 \mathrm{~mm}$ and $z=20$ $\mathrm{mm}$ for the two-layered laminates with $h_{m}=1,3$ and $5 \mathrm{~mm}$ at frequency $f=50 \mathrm{~Hz}$. A nonlinear relationship between tip deflection and $\mathrm{AC}$ electric field amplitude is observed. Fig. 3 shows the induced magnetic field $B_{\text {in }}$ versus $E_{0}$ for the two-layered laminates with $h_{m}=1,3$ and $5 \mathrm{~mm}$ at $f=$ $50 \mathrm{~Hz}$, obtained from the FEA and test.

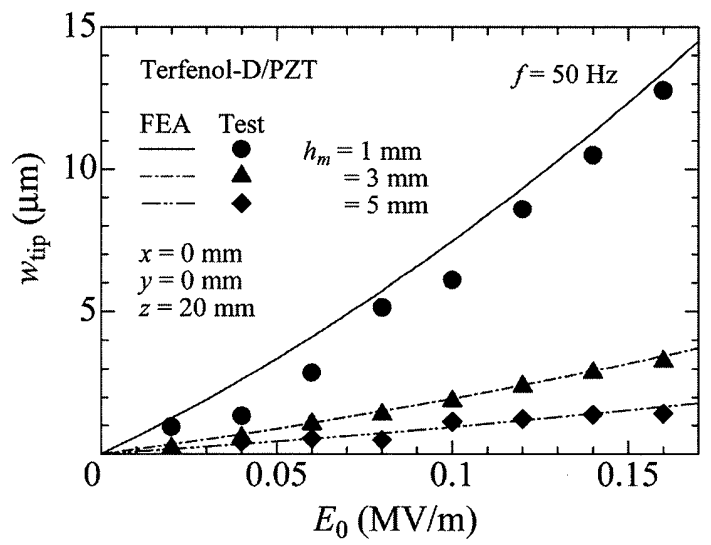

Fig. 2 Tip deflection versus AC electric field amplitude for two-layered magnetostrictive/piezoelectric laminates

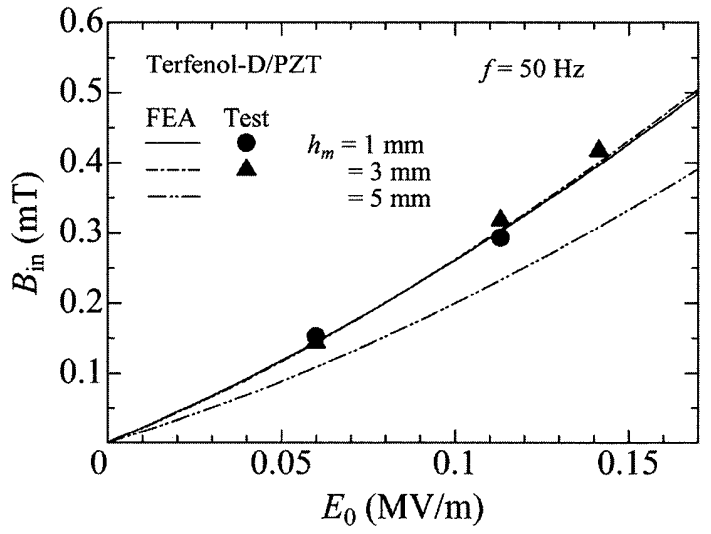

Fig. 3 Induced magnetic field versus AC electric field amplitude for two-layered laminates

\section{References}

1) F. Narita, Y. Shindo, M. Mikami, Acta Mater. 53, 4523-4529 (2005)

2) Y. Shindo, F. Narita, K. Mori, T. Nakamura, J. Mech. Mater. Struct. 4, 941-949 (2009).

3) Y. Shindo, K. Mori, F. Narita, Acta Mech. 213, 253261 (2010) 